\title{
Perceptually-Inspired Computing
}

\author{
Keynote
}

\author{
Ming C. Lin \\ Department of Computer Science \\ University of North Carolina at Chapel Hill and Tsinghua University \\ lin@cs.unc.edu
}

\begin{abstract}
Human sensory systems allow individuals to see, hear, touch, and interact with the surrounding physical environment. Understanding human perception and its limit enables us to better exploit the psychophysics of human perceptual systems to design more efficient, adaptive algorithms and develop perceptually-inspired computational models.

In this talk, I will survey some of recent efforts on perceptually-inspired computing with applications to crowd simulation and multimodal interaction. In particular, I will present data-driven personality modeling based on the results of user studies, exampleguided physics-based sound synthesis using auditory perception, as well as perceptually-inspired simplification for multimodal interaction. These perceptually guided principles can be used to accelerating multi-modal interaction and visual computing, thereby creating more natural human-computer interaction and providing more immersive experiences. I will also present their use in interactive applications for entertainment, such as video games, computer animation, and shared social experience. I will conclude by discussing possible future research directions.
\end{abstract}

Keywords - perceptually-inspired computing; human perceptual systems; crowd simulation; multimodal interaction; humancomputer interaction; entertainment; video games; computer animation

\section{BIOGRAPHY}

Ming C. Lin is currently John R. \& Louise S. Parker Distinguished Professor of Computer Science at the University of North Carolina (UNC), Chapel Hill and an honorary Chair Professor (Yangtze Scholar) at Tsinghua University in China. She obtained her B.S., M.S., and Ph.D. in Electrical Engineering and Computer Science from the University of California, Berkeley. She received several honors and awards, including the NSF Young Faculty Career Award in 1995, Honda Research Initiation Award in 1997, UNC/IBM Junior Faculty Development Award in 1999, UNC Hettleman Award for Scholarly Achievements in 2003, Beverly W. Long Distinguished Professorship 2007-2010, Carolina Women's Center Faculty Scholar in 2008, UNC WOWS Scholar 20092011, IEEE VGTC Virtual Reality Technical Achievement Award in 2010, and nine best paper awards at international conferences.

She is a Fellow of ACM and IEEE. Her research interests include physically-based modeling, virtual environments, sound rendering, haptics, robotics, and geometric computing. She has (co-)authored more than 250 refereed publications in these areas and co-edited/authored four books. She has served on over 130 program committees of leading conferences and co-chaired dozens of international conferences and workshops.

She has been the Editor-in-Chief of IEEE Transactions on Visualization and Computer Graphics (2011-2014). She is a member of 6 editorial boards, and a guest editor for over a dozen of scientific journals and technical magazines. She also has served on several steering committees and advisory boards of international conferences, as well as government and industrial technical advisory committees. 